Développement des territoires et communication : politiques et pratiques à l'œuvre

\title{
Territoires, proximité et espace public
}

Territories, Proximity, and Public Space

\section{Alain Girod}

\section{(2) OpenEdition}

\section{Journals}

Édition électronique

URL : http://journals.openedition.org/edc/97

DOI : $10.4000 /$ edc. 97

ISSN : 2101-0366

Éditeur

Université Lille-3

\section{Édition imprimée}

Date de publication : 1 décembre 2003

ISSN : 1270-6841

Référence électronique

Alain Girod, «Territoires, proximité et espace public », Études de communication [En ligne], 26 | 2003,

mis en ligne le 09 octobre 2008, consulté le 03 mai 2019. URL : http://journals.openedition.org/edc/97 ; DOI : 10.4000/edc.97

Ce document a été généré automatiquement le 3 mai 2019.

(c) Tous droits réservés 


\title{
Territoires, proximité et espace public
}

\author{
Territories, Proximity, and Public Space
}

Alain Girod

1 Depuis quelques années, semble se dessiner une inflexion des discours politiques et institutionnels relatifs au développement des territoires. Le thème de la "proximité " tend en effet à apparaître comme le fondement de l'action politique, comme en témoignent, pour ne prendre que quelques exemples très récents, la loi sur la «démocratie de proximité " du 27 février 2002, la loi constitutionnelle sur la décentralisation votée le 17 mars 2003, ou encore les discours des élus locaux, en tout cas dans l'agglomération lyonnaise.

2 Un deuxième thème, fortement lié au premier, structure également les discours politiques et institutionnels : celui de la « démocratie locale » et de la « consultation des habitants " censé permettre l'émergence d'une nouvelle citoyenneté. La «France d'en bas » est aujourd'hui parée de toutes les vertus et la politique semble devoir se réduire de plus en plus dans le temps et dans l'espace, avec pour seuls horizons le quotidien et le quartier.

3 Ce «localisme» politique n'est certes pas totalement nouveau: on en trouve des manifestations dès la fin des années 1960 (Groupes d'action municipale), et plus encore à partir du début des années 1980, avec la généralisation progressive des références au « quartier » considéré comme le lieu pertinent de l'action publique. Mais il nous apparaît qu'un changement qualitatif est intervenu depuis quelque temps, d'une part parce que la "proximité » est devenue un thème prégnant dans tous les discours politiques et institutionnels, et d'autre part parce qu'elle tend à se substituer aux véritables enjeux politiques. La tendance évoquée dès 1995 par Jean-François Tétu à propos de la communication publique semble donc bien se confirmer et s'accentuer: "Si la démocratie est devenue clairement médiatique, cette médiatisation tend de plus en plus à 
reposer non pas sur l'intérêt général de la nation, mais sur la priorité du local » (Tétu, 1995).

4 On ne peut manquer, à partir de là, de s'interroger sur les conséquences de cette évolution sur le fonctionnement de l'espace public. En effet, la "proximité », le souci louable de rapprocher les représentants des représentés, la volonté - réelle ou feinte d'améliorer la démocratie, ne risquent-ils pas d'une part de remettre en cause la légitimité du politique, et d'autre part d'accentuer les effets pervers des relations entre les individus et l'État-providence? Les discours et les pratiques que nous avons évoqués ci-dessus ne tendent-ils pas à réduire le champ d'intervention des citoyens à des questions limitées au territoire du quartier ou aux services que le contribuable est en droit d'attendre d'une collectivité locale ? Cette espèce de coup de force visant à transformer l'espace public, qui est un lieu symbolique de débat, en un lieu géographique, en un territoire, est lourd de conséquences, en particulier parce qu'il focalise l'espace public sur des approches privées ou semi privées (en ce sens qu'elles sont relatives non pas à l'intérêt général ou à des intérêts collectifs idéologiquement identifiés, mais à des intérêts individuels ou localistes).

5 Cette problématique rejoint en partie certaines thèses développées par Habermas dans «L'espace public» (Habermas, 1978), en particulier pour ce qui est d'une certaine « reféodalisation » de la société qui serait induite par la transformation de l'État libéral en «État social » ou en « État providence ». Pour autant, nous ne sous-estimons pas la portée des nombreuses critiques qui ont pu être adressées à Habermas, d'abord par lui-même, ensuite par différents auteurs comme Paul Beaud, Patrice Flichy, Pierre Moeglin, ou Bernard Miège.

6 En effet, près de trente ans après avoir publié «L'espace public », Habermas lui-même a proposé «trois révisions » de son analyse des transformations structurelles de la sphère publique $^{1}$. D'une part, ayant pris conscience des «faiblesses de la pensée hégélianomarxiste dans son concept de totalité ", il a "pris (ses) distances vis-à-vis de cette conception ». D'autre part, il a admis avoir sous-estimé la «capacité de résistance, et surtout le potentiel critique d'un public de masse pluraliste et largement différencié, qui déborde les frontières de classe dans ses habitudes culturelles ». Enfin, Habermas semble finalement admettre que le "pluralisme d'intérêts irréconciliables » qui, dans le cadre de l'État social, luttent entre eux et contre l'administration pour imposer des compromis, constitue un obstacle sérieux à sa théorie.

7 Cette troisième " autocritique » d'Habermas rejoint une réflexion commune à la plupart de ceux qui, depuis une trentaine d'années, réfléchissent à propos de l'espace public. De nombreux chercheurs considèrent en effet que, pour « réactiver » le concept « d'espace public», il convient d'y apporter des modifications substantielles, notamment « d'accepter l'idée que l'espace public est un espace pour le moins conflictuel (certains ajoutant : où s'exerce - notamment - la domination de la classe bourgeoise sur les autres classes)» (Miège, 1989). De même il existe un relatif consensus pour reconnaître dans «l'espace public » une conception "manipulatoire » des médias de masse et une vision assez simpliste de leurs effets dans une société dominée par la consommation de masse.

8 Ce n'est évidemment pas le lieu d'en discuter ici. Mais il nous semble tout de même indispensable de rappeler brièvement les perspectives stimulantes ouvertes par Bernard Miège pour qui l'espace public «se perpétue [...] s'élargit (toutes les classes et catégories sociales y prennent part, mais à des titres divers); il voit ses fonctions s'étendre régulièrement $[. .$.$] ; et il a tendance à se fragmenter » (Miège, 1995). C'est à partir de ce$ 
dernier élément, qui nous semble particulièrement éclairant, que nous nous demanderons si la "proximité» n'a pas notamment comme effet pervers une fragmentation de plus en plus poussée de l'espace public, avec des conséquences lourdes en termes de démocratie et de débat public que nous essaierons de mettre en évidence.

9 Nous nous proposons donc d'articuler notre contribution autour de trois axes: une analyse des discours institutionnels sur les territoires et la proximité, en particulier à partir de l'exposé des motifs de la loi sur la démocratie de proximité ; une analyse de la communication et des pratiques de «démocratie locale » de deux collectivités locales significatives (Villeurbanne et la Communauté Urbaine de Lyon); enfin une réflexion plus théorique sur les évolutions de l'espace public induites par la territorialisation de l'action publique.

\section{Genèse de la « proximité »}

10 Pour comprendre la genèse de la "proximité ", il est nécessaire d'en analyser les trois sources principales: la «crise des banlieues» et la «politique de la ville»; la « décentralisation »; la désaffection des citoyens pour la politique, caractérisée, depuis le milieu des années 1980, par des taux d'abstention record lors des différentes élections. Nous n'avons hélas pas la place de développer ces trois éléments comme ils devraient l'être. Nous nous contenterons donc d'indiquer que depuis 1981, l'évolution de la situation politique et sociale, les problèmes existant dans un certain nombre de quartiers défavorisés et la crise de la démocratie représentative ont conduit les pouvoirs publics, aussi bien au niveau national qu'au niveau local, à considérer la « proximité » comme une réponse pertinente permettant d'asseoir une nouvelle légitimité pour les représentants du peuple, dans le cadre d'une espèce de « sacralisation » du citoyen².

11 Il faut également considérer un phénomène social majeur : le développement depuis les années 1960 de la notion "d'opinion publique »" qui est devenue, en raison notamment de la multiplication des sondages d'opinion, un mode de légitimation indispensable de tout discours et de toute action publique, et ce d'autant plus que les électeurs boudent les urnes.

12 Au-delà de cette logique sociale générale, il est intéressant d'analyser plus précisément comment la «démocratie de proximité » a été institutionnalisée par la loi du 27 Février 2002.

13 Le texte évoque notamment une "exigence de proximité de plus en plus forte » qui " témoigne du souhait des Français de participer à la réflexion sur les décisions locales et à la définition des projets qui les concernent au quotidien ». C'est la commune qui est considérée comme « le lieu privilégié d'apprentissage et d'exercice de la démocratie », la « démocratie de proximité » ayant pour objet de favoriser « l'expression de la citoyenneté au niveau local ». Il s'agit donc de mieux associer « l'ensemble des citoyens aux décisions locales ", de créer les conditions de la "participation des habitants au débat public ", l'outil essentiel de cette participation étant les « conseils de quartier », dont la création est rendue obligatoire dans les communes de plus de 80000 habitants «pour chacun des quartiers qui les constituent». Les conseils de quartier sont définis comme des " instances consultatives » permettant d'associer élus, habitants et associations "pour traiter de toute question intéressant le quartier». L'objectif général est de «mieux prendre en compte les questions de proximité », de « veiller à l'information des habitants 
et de favoriser leur implication dans la vie locale ». Il est enfin indiqué que « promouvoir la démocratie de proximité » implique "l'amélioration de l'accès aux services de proximité par la création de mairies annexes ». Ces annexes de la mairie sont rendues obligatoires dans les communes de plus de 100000 habitants. Cette loi vise donc, en résumé, «l'amélioration effective de l'implication des habitants dans le processus de décision local ».

Cette loi fonde sa légitimité interne sur les destinateurs que sont les «citoyens", les "Français », mais elle désigne souvent ceux-ci par des vocables qui ne renvoient plus à une appartenance générale, mais à une appartenance locale comme « habitants » ou à un caractère fonctionnel, « usagers » (en l'espèce, « usagers » des services municipaux).

C'est en réalité «le quartier» qui devient le lieu privilégié de la démocratie, comme en atteste l'obligation de créer des «mairies de quartier» offrant des «services de proximité » dans les grandes villes. On peut également remarquer que si l'horizon géographique est limité au quartier, l'horizon temporel s'arrête au " quotidien », puisqu'il s'agit d'associer les «habitants» à la «définition des projets qui les concernent au quotidien ».

16 Il est enfin intéressant d'analyser la conception de la "démocratie de proximité » que sous-tend cette loi. Il apparaît en effet que la « démocratie de proximité » repose sur trois éléments : les " conseils de quartier ", l'information des habitants par les élus, et la mise en place dans les grandes villes de «mairies annexes» offrant des «services publics de proximité ».

17 L'élément le plus important est évidemment la mise en place des « conseils de quartier ». Ces instances, qui rassemblent élus, habitants et associations, sont purement consultatives. Elles ont une compétence strictement limitée à « toute question intéressant le quartier » et la loi prend grand soin d'encadrer leur action dans la mesure où elle précise: «Les conseils de quartier ne sauraient constituer un organe concurrent du conseil municipal. Ils interviendront, de façon complémentaire, en amont du processus décisionnel ».

18 La "démocratie de proximité », selon la loi du 27 février 2002, repose également sur l'information des habitants par les élus. Cette « mission première » n'est évidemment pas négligeable. Mais ce souci louable de mieux informer les habitants n'a rien de très novateur, d'autant qu'il reste au niveau de la déclaration d'intention. On peut au surplus se demander si cette information, limitée aux questions de proximité et administrée par les seuls élus, peut avoir d'autre finalité que de justifier les décisions prises ou la pertinence des projets des communes.

19 Enfin, le troisième pilier de la « démocratie de proximité » serait constitué, aux termes de la loi, par la mise à disposition des « usagers » de «services publics de proximité » dans les « mairies de quartier », et ce pour les villes de plus de 100000 habitants.

\section{Villeurbanne et le « Grand Lyon »}

Comme nous l'avons déjà indiqué, cette loi relative à la "démocratie de proximité " entérine et institutionnalise des discours et des pratiques qui existent depuis déjà un certain nombre d'années, notamment dans l'agglomération lyonnaise, et plus précisément dans la ville de Villeurbanne ${ }^{4}$ qui a mis en place des conseils de quartier depuis 1995. 
21 Disons simplement, pour ce qui concerne Villeurbanne, qu'il s'agit d'un territoire emblématique, à bien des égards, de ce que nous avons précédemment évoqué en termes de développement social des quartiers, de décentralisation et de crise de la démocratie représentative.

Cela permet de mieux comprendre pourquoi la "proximité » et la « démocratie locale » constituent un axe majeur de la communication et des pratiques municipales. C'est ainsi qu'au-delà des conseils de quartier, les élus organisent à intervalles très réguliers des "visites de quartier» au cours desquelles ils rencontrent les habitants sur un itinéraire rendu public par voie de tracts; de même des "permanences de quartier » ont lieu au moins un samedi matin par trimestre pour permettre aux gens de venir exposer leurs problèmes. Et la municipalité a même décidé d'aller plus loin encore, puisqu'elle a commandité un véritable sondage d'opinion, dans chaque quartier, doublé d'un questionnaire publié dans "Viva» de Février 2003 (magazine municipal) pour mieux mesurer les attentes de la population. Le texte de présentation de cette enquête, également publié dans "Viva", nous semble assez illustratif du discours municipal: «Depuis 1995 et la mise en route progressive de la démocratie locale, les habitants de Villeurbanne sont régulièrement consultés dans les conseils de quartier, dans les réunions publiques que nous organisons, ou au cours des visites que leur font les élus, sur les projets propres à leur quartier. Loisirs, environnement, urbanisme, Villeurbanne avance, toujours à l'avant-garde, tout en protégeant cependant son identité, sa mémoire. L'équipe municipale met en place des actions dans tous ces domaines. Mais vous, qu'en pensez-vous?».

D'une façon plus générale, on peut constater que l'ensemble de la communication municipale, que ce soient le magazine, le site internet, les affiches, les plaquettes, les tracts, etc., est très fortement imprégné par le thème de la proximité, des quartiers, de la démocratie locale. Et l'accent mis sur les quartiers est tel qu'un supplément à "Viva » propre à chaque quartier est réalisé chaque mois. Il faut également signaler que deux adjoints au maire (sur quinze) sont chargés de la «proximité », l'un de la «population et des services publics de proximité », l'autre de la « démocratie locale ».

Mais, bien évidemment, la "proximité » dont se réclament les élus locaux ne peut s'analyser uniquement à partir de ses manifestations explicites. Il faut aussi prendre en compte dans la communication publique locale toute une mise en scène, toute une stratégie éditoriale visant à privilégier l'individu ordinaire, l'action et la parole singulières. De ce point de vue, il nous apparaît que la communication municipale comme d'ailleurs la communication politique nationale - a intégré, avec un temps de retard, une évolution que l'on a pu constater dans les médias, et notamment à la télévision, depuis le début des années 1990. Cette évolution a été remarquablement analysée par Dominique Mehl dans son ouvrage intitulé « La télévision de l'intimité » où elle souligne notamment : "l'irruption de l'expérience profane et du témoignage privé sur la scène publique » (Mehl, 1996).

Si l'on considère d'une façon diachronique la communication publique locale, on peut, comme l'a fait Isabelle Pailliart, repérer plusieurs périodes bien différentes les unes des autres. Pour elle, « la décennie 1970 attribuait à l'information municipale une capacité fondatrice à animer le tissu local [...], alors que la décennie suivante rapproche l'information et la communication locales d'autres modèles : la communication politique et la communication des organisations ", en empruntant à la communication politique « la valorisation du pouvoir local » et à la communication d'entreprise «ses possibilités 
de création d'une identité ainsi que l'émergence d'un nouveau mode de gestion des services urbains locaux » (Pailliart, 1993).

Nos propres recherches nous conduisent à poser l'hypothèse que la décennie 2000 est caractérisée par l'émergence d'un nouveau modèle, «la communication de proximité ", tout en précisant deux éléments importants: d'une part, ce nouveau modèle ne se substitue pas à celui de la communication politique et à celui de la communication des organisations, qui restent à certains égards une dimension essentielle de la communication publique locale. D'autre part, la «communication de proximité » ne constitue pas un simple "retour du local», mais un paradigme nouveau, qui reste à analyser dans le détail, mais qui s'inscrit dans un phénomène général évoqué par Dominique Mehl : «il se caractérise par une nouvelle forme d'articulation de la parole privée et de la parole publique. La parole privée ne vient plus seulement illustrer un propos ou accompagner une démonstration. Elle vaut en elle-même comme parole publique, c'est-à-dire comme parole susceptible de nourrir le débat collectif, de s'intégrer à l'espace public »(Mehl, 2002).

Du coup, même si la promotion directe de l'action municipale - ou communautaire demeure présente, elle semble de plus en plus devenir secondaire, ou plutôt elle tend à être mise en valeur indirectement, par une espèce d'effet de miroir, par la mise en scène d'habitants heureux, qui réussissent, qui portent une parole positive. Et c'est à partir de là que peut être réactivée la vieille formule de l'homme politique qui se présente comme "celui qui vous ressemble, celui qui vous rassemble». C'est ainsi que la ville de Villeurbanne, au début de l'année 2003, a adopté ce «nouveau » slogan : «Villeurbanne, la ville qui nous ressemble, la ville qui nous rassemble ", décliné par voie d'affiches, de brochures, et particulièrement utilisé depuis dans tous les documents relatifs à la démocratie locale.

Notre recherche sur le magazine «Viva » a porté sur 12 numéros publiés entre janvier 2002 et février $2003^{5}$. Nous avons d'abord pu constater que, contrairement aux pratiques antérieures, notamment dans les années 1980, les photographies du maire et des élus sont devenues marginales, alors que l'essentiel de l'iconographie est consacré à des individus inconnus ou à de petits groupes d'habitants. En moyenne, il n'y a guère que $10 \%$ des photographies qui représentent des élus, $80 \%$ montrant des habitants, et $10 \%$ seulement d'autres objets (bâtiments publics, paysages etc.), chaque numéro de « Viva » comportant environ 50 à 60 photographies. On a donc là un premier signe d'une stratégie de communication visant à valoriser les habitants et à montrer que la municipalité est «proche des gens ».

29 Si l'on prend maintenant en considération l'ensemble des 50 à 60 articles publiés dans chaque numéro de "Viva", à l'exception de la tribune libre des groupes politiques, rendue obligatoire par la loi, et de "l'information service » (2 pages), on peut remarquer que le tiers environ de ces textes organise une mise en scène d'habitants «lambda », un second tiers relevant de la promotion directe d'initiatives municipales, le troisième tiers étant consacré à la valorisation d'initiatives non municipales mais se déroulant sur le territoire de la commune, et permettant de donner une image positive de la ville. Mais il faut préciser que ces deux derniers tiers sont souvent illustrés par des photographies de citoyens ordinaires et/ou construits autour d'une parole individuelle.

C'est ainsi, par exemple, que la commémoration de l'appel du 18 juin 1940 sera traitée non pas à partir du discours du maire, mais à partir de la lecture de l'appel par une petite fille, Eléonore, dans un article intitulé : «18 juin : l'appel d'Eléonore » (Viva, juillet-août 
2002). Ou encore le portage des repas à domicile pour les personnes âgées, organisé par la ville, sera traité en bonne partie à partir du témoignage d'une bénéficiaire et d'une « porteuse de repas », photographie à l'appui (Viva, mars 2002).

Quant à la mise en scène d'habitants ordinaires, elle vise d'une certaine manière à montrer qu'à Villeurbanne tous les habitants sont «extraordinaires", et donc que les élus, qui leur ressemblent, qui sont proches d'eux, le sont aussi. Car bien sûr, s'il faut avoir réalisé quelque chose de particulier pour avoir les honneurs de «Viva », on constate que les motifs de cet hommage d'un jour ou plutôt d'un mois, peuvent être très divers et parfois ténus. C'est ainsi que vont se retrouver pèle-mêle, dans la catégorie des « citoyens d'honneur " des centenaires, des anciens combattants, une rescapée du génocide des arméniens, des responsables sportifs ou associatifs, des peintres du dimanche, des militants humanitaires, des retraités, des membres des conseils de quartier, des ecclésiastiques, des délégués de classe, des chômeurs, le jeune président de la M.J.C., un historien amateur, un "télétravailleur», etc. Bel inventaire à la Prévert dans lequel chacun ou presque peut se reconnaître, dans lequel chacun a une chance de se retrouver un jour. Voilà une représentation presque caricaturale de la "France d'en bas ", enfin reconnue et admirée grâce au journal municipal...

Si Villeurbanne s'est investie dans la « démocratie de proximité » longtemps avant la loi du 27 Février 2002, on ne peut pas en dire autant de la Communauté Urbaine de Lyon qui, avant le renouvellement de 2001, ne s'en préoccupait pas du tout. Il est vrai que la problématique du « Grand Lyon » est toute différente de celle d'une commune. En effet, la COURLY n'est pas à proprement parler une " collectivité territoriale ", puisqu'elle n'est pas reconnue comme telle par la Constitution. Il s'agit en fait, juridiquement, d'un "établissement public de coopération intercommunale», doté d'une fiscalité propre, mais dont les dirigeants - les «conseillers communautaires» - ne sont pas élus au suffrage universel, puisqu'ils sont désignés par les conseils municipaux des communes membres. Il y a donc, dès le départ, un déficit de démocratie dans les structures intercommunales.

Mais le paradoxe, c'est que ces structures peu démocratiques et peu responsables devant la population, exercent, par délégation des communes qui en sont membres, des compétences extrêmement importantes en matière d'urbanisme, de transports en commun, d'aménagement urbain, de développement économique, etc. Parmi celles-ci se trouvent notamment des " compétences de proximité », comme l'enlèvement des ordures ménagères, l'assainissement, la propreté, la voirie, etc. On se trouve donc face à une énorme "technostructure ", avec des milliers d'agents, un budget annuel de plusieurs milliards d'euros, des moyens techniques considérables, des compétences essentielles pour la vie quotidienne de tout un chacun, et en même temps avec des élus que personne n'identifie comme tels, avec des pratiques technocratiques et qui se soucient peu des habitants.

Si, jusqu'en 2001, le thème de la proximité était totalement absent du discours du « Grand Lyon ", il est aujourd'hui omniprésent aussi bien dans les propos du président Gérard Collomb, également maire de Lyon, que dans les supports de communication. C'est ainsi, pour ne prendre qu'un seul exemple, que le magazine de la Communauté Urbaine de Lyon, intitulé "Grand Lyon magazine », reprend sur la « une », de façon très visible, le slogan de la COURLY, «Voir grand et être proche ». Cette antithèse renvoie au paradoxe que nous évoquions ci-dessus et montre, de façon assez habile, comment le «Grand Lyon » essaie de conjuguer, dans sa communication, sa forte ambition de jouer un rôle 
majeur dans la région, l'Europe et le monde et son souci affiché de gérer l'agglomération en tenant le plus grand compte des demandes des habitants.

Le numéro de «Grand Lyon Magazine » de janvier-février 2003, premier numéro de la nouvelle formule, est à cet égard très significatif. On peut en effet voir dans cette publication, y compris à la "une ", les deux faces de Janus, à savoir d'une part le grand projet «Lyon Confluence » et d'autre part, le projet de «charte de la participation », le premier occupant l'essentiel de la « une », l'éditorial du Président et deux pages de texte, le second devant se contenter d'un appel de « une » et de trois-quarts de page. Mais il faut aussi noter que, dans l'éditorial du Président, dès le premier paragraphe, la proximité est présentée comme un des trois objectifs majeurs de la COURLY : «Améliorer la qualité de votre cadre de vie, être plus près de vos préoccupations et faire du Grand Lyon une métropole rayonnante, tels sont les objectifs que nous nous fixons [...]. Le projet de Lyon Confluence qui vous est présenté dans ce numéro répond à cette triple exigence. Cette opération emblématique du développement de l'agglomération est un des projets majeurs d'aménagement de centre-ville en Europe ».

Quant à la " charte de la participation ", elle se fixe pour objectif de "développer une démocratie plus participative [...]. Elle précisera les orientations et les principaux engagements du 'Grand Lyon' pour une meilleure association des citoyens [...]». Pour cela, la Communauté Urbaine s'engage par exemple à améliorer la diffusion de documents d'information sur son activité, à organiser des expositions sur ses projets dans les lieux publics et à ouvrir le dialogue sur ceux-ci. La participation s'incarnera également dans différents dispositifs ouverts aux associations: la "conférence d'agglomération de l'habitat ", la commission consultative "Urbanisme et déplacements ", le schéma de développement économique et la " commission consultative des services publics locaux ».

La " démocratie de proximité », la " participation », la " concertation », la " consultation des habitants " sont donc omniprésents dans toute la communication émanant du "Grand Lyon ». Le "Grand Lyon », qui a beaucoup de retard sur ce terrain, cherche à apparaitre plus proche de ses habitants en s'affichant davantage à leur écoute, en prenant davantage en compte leur parole, en insistant sur sa volonté « d'informer les habitants le plus complètement possible ». On peut néanmoins se demander, avec Christian Le Bart, si la communication publique locale ne constitue pas «une contribution ambiguë à la démocratie locale ». Selon lui en effet, « la légitimité unanimement conférée aux bulletins municipaux résulte d'un intense travail accompli par les élus et les professionnels de la communication pour assimiler, sur le mode de l'évidence qu'il n'y aurait presque plus lieu de problématiser, l'information à la démocratie » (Le Bart, 2000).

\section{Vers un nouveau déclin de l'espace public?}

Il reste à réfléchir, d'une façon un peu plus conceptuelle, à propos des effets de la " proximité » sur le fonctionnement de l'espace public. Nous sommes hélas contraints, faute de place, de le faire de façon sommaire.

Nous souhaitons tout d'abord souligner le fait que la démocratie a encore beaucoup de progrès à effectuer et qu'il est tout à fait légitime de rechercher des solutions à ce grave problème qu'est le divorce apparent entre de très nombreux citoyens et la politique comprise comme le débat public rationnel et/ou idéologique sur des questions d'intérêt général. 
40 Mais il nous paraît tout aussi légitime de se demander si les solutions envisagées, en l'espèce la «proximité » et la "démocratie locale » ou "participative », sont totalement pertinentes. Au fond, la question qui se pose est de savoir si l'approfondissement de la démocratie « directe " peut être une réponse à la crise de la démocratie représentative ou s'il ne conviendrait pas de repenser les relations entre ces deux formes aussi imparfaites l'une que l'autre et dont le rapport dialectique ne renvoie certainement pas au principe des vases communicants, mais bien plutôt à l'articulation entre le singulier et le collectif. Nous formulons donc l'hypothèse que, compte tenu des discours et des pratiques que nous avons analysés, la "proximité » et la " démocratie participative » ne peuvent ni se substituer à la démocratie représentative ni redonner aux citoyens le sens du débat politique. Et comme l'indique très bien Claude Sorbets, «plus la distance s'accroît entre les citoyens ordinaires et les décideurs, entre les participants à la comédie du pouvoir, d'un pouvoir qui se diffuse et se dilue dans des dispositifs, appareils ou montages d'actions, et plus on parle de transparence, de proximité et de responsabilité répartie et prétendue ramenée vers ceux auxquels les décisions sont supposées destinées » (Sorbets, 2002).

41 À l'issue de cette étude, on peut essayer d'avancer quelques pistes pour essayer de répondre un peu plus précisément à notre problématique initiale telle qu'elle nous était suggérée par Bernard Miège : quelles sont les incidences de la proximité en termes de fragmentation de l'espace public?

Disons, pour aller vite, que la "proximité », le repli sur le quartier, la démocratie locale trop souvent manipulatrice risquent fort de conduire, volens nollens, à une accentuation de la fragmentation de l'espace public et à la multiplication d'espaces parcellisés sans véritables relations les uns avec les autres. Car la proximité réduit tellement l'espace public que, d'une certaine façon, elle le nie. Cette situation, dans une société largement dominée par les médias de masse et les dispositifs communicationnels de toutes sortes mis en oeuvre par les institutions sociales de toutes natures (entreprises, collectivités locales, État, etc.), peut avoir de graves conséquences : aggravation de la domination des individus les plus défavorisés, approfondissement des inégalités culturelles et sociales dans la participation au débat public, recul des solidarités, et surtout renforcement d'un consensus politique et social artificiel fondé sur le consumérisme et l'individualisme. Disons enfin qu'un autre avenir est sans doute possible, à condition que la proximité ne se substitue pas au débat politique et idéologique de fond, à condition que la démocratie locale aille de pair avec une profonde démocratisation des processus décisionnels à tous les niveaux, à condition que les représentants du peuple, les partis politiques et les citoyens eux-mêmes aient le courage et la volonté d'élaborer des choix alternatifs.

\section{BIBLIOGRAPHIE}

Le Bart, C., (2000), « Les bulletins municipaux : une contribution ambiguë à la démocratie locale », in : Hermès, n²6-27 («www.démocratie locale.fr »), CNRS Éditions, Paris.

Mehl, D., (1996), La télévision de l'intimité, Le Seuil, Paris. 
Mehl, D., (2002), « Télévision de l'intimité et espace public », in : La vie privée à l'heure des médias, Presses Universitaires de Bordeaux.

Miège, B., (1989), La société conquise par la communication, Presses Universitaires de Grenoble.

Miège B., (1995), « L'espace public : perpétué, élargi et fragmenté », in : Pailliart I. (dir.), L'espace public et l'emprise de la communication, ELLUG, Grenoble.

\section{NOTES}

1. Préface à l'édition de 1990.

2. Cf. notamment Rosanvallon, 1992.

3. Cf. Bourdieu, 1984.

4. Villeurbanne, qui jouxte Lyon, est située à l'est de l'agglomération. Avec 127000 habitants, c'est la deuxième ville $\mathrm{du}$ " Grand Lyon ».

5. «Viva » est un magazine d'un format $23 \mathrm{~cm} \times 30 \mathrm{~cm}$, qui compte 36 pages, entièrement en quadrichromie, dont les articles ne sont pas signés, à l'exception de l'éditorial du maire en page 3. Cette revue ne comporte aucune publicité.

\section{RÉSUMÉS}

Le thème de la " proximité » et de la « démocratie locale » est aujourd'hui omniprésent dans les discours politiques et institutionnels. Il est donc intéressant d'analyser ces discours et les pratiques qui s'en réclament en essayant de préciser leur genèse : crise des "banlieues", décentralisation, et crise de la démocratie représentative. Et, à partir de l'analyse de quelques textes fondamentaux (loi de décentralisation de 2003, loi sur la "démocratie de proximité » de 2002) ainsi que des dispositifs communicationnels mis en place dans la ville de Villeurbanne et dans la Communauté Urbaine de Lyon, il convient de s'interroger sur les effets pervers de la " proximité » sur le fonctionnement de l'espace public contemporain, notamment à partir des travaux d'Habermas et de Bernard Miège, en se demandant notamment si la "proximité » ne risque pas de conduire à une nouvelle fragmentation de l'espace public.

The theme of the proximity of local democracy is today omnipresent in political and institutional speeches. It is therefore interesting to analyze these speeches and the practical experiences they evoke by specifying their genesis in a crisis of the suburbs, decentralization, or a crisis of representative democracy. Based on the analysis of a number of foundational texts (the Decentralization Act of 2003 and the Proximity and Democracy Act of 2002) as well as communicational devices set up in the city of Villeurbanne and in the Urban Community of Lyon, we reflect on the perverse effects of proximity on the operations of the public sphere, particularly thanks to publications of Habermas and Bernard Miège, and ask whether proximity may not run the risk of bringing about a new fragmentation of the public sphere. 
INDEX

Mots-clés : développement territorial, discours politique, espace public

Keywords : territorial development, political speech, public sphere

\section{AUTEUR}

\section{ALAIN GIROD}

Alain Girod - Institut de la communication - Université de Lyon 2 